\title{
Managing environmental lead in Broken Hill: a public health success
}

\section{Frances Boreland $^{\mathrm{A}, \mathrm{C}}$, Margaret S. Lesjak ${ }^{\mathrm{A}}$ and David M. Lyle $\mathrm{B}^{\mathrm{B}}$}

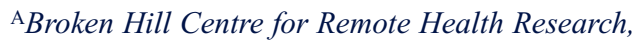
joint initiative of the University of Sydney and

Greater Western Area Health Service

${ }^{\mathrm{B} B r o k e n ~ H i l l ~ U n i v e r s i t y ~ D e p a r t m e n t ~ o f ~ R u r a l ~ H e a l t h, ~}$ University of Sydney

${ }^{\mathrm{C} C o r r e s p o n d i n g ~ a u t h o r . E m a i l: ~ f b o r e l a n d @ ~}$ gwahs.health.nsw.gov.au

\begin{abstract}
Objectives: To describe localityspecific changes in blood lead levels of 1-4-yearold children in Broken Hill, NSW between 1991 and 2007. Methods: Annual age-sex standardised mean blood lead levels, blood lead screening clinic attendance rates and lead-dust levels for five leadrisk zones were calculated from routinely collected data. Results: Blood lead levels were similar in all localities in 2002, 2003, 2005 and 2006, after having been consistently higher in localities with highest environmental lead since 1991. Conclusions: Combining health promotion with a targeted clean-up has reduced the effect of locality on blood lead levels. Results are consistent with reduced contamination due to effective soil stabilisation and storm-water control.
\end{abstract}

"For whoever has, to him more shall be given; and whoever does not have, even what he has shall be taken away from him" is an apt description of many health programs, in that while everyone benefits, those who have least need of the program tend to benefit most, and those who need the program most, often do not benefit at all. ${ }^{1}$ This paper, however, describes the outcomes of a program for which this has not been the case. We describe the trend for locality to have a reduced impact on blood lead levels of young children living in Broken Hill, New South Wales (NSW), first observed in 2002. ${ }^{2}$

Lead has been mined in Broken Hill since 1884. Lead poisoning was evident among the early miners and their families, but was seen as mainly an occupational health problem. ${ }^{3}$
This was reinforced by a survey of school-aged children in 1982, which found all had blood lead levels below $40 \mu \mathrm{g} / \mathrm{dL}$, the then level of concern in Australia. ${ }^{4,5}$ However, a subsequent study of apparently healthy Broken Hill dogs found blood lead levels similar to levels found in dogs in the town of Port Pirie, South Australia, which has an active lead smelter. ${ }^{6}$ Local concern was further increased in Broken Hill by the recommissioning of open-pit mining in the centre of town, a drought in the late 1980s and the birth of three babies with delayed visual maturation (usually caused by exposure to high lead levels in utero) between 1988 and 1990. ${ }^{7}$ A survey of 1-4-year-old Broken Hill children in 1991 found $86 \%$ had blood lead levels of $10 \mu \mathrm{g} / \mathrm{dL}$ or above (the current level of concern with regard to health effects) and 38\% had very high lead levels of $20 \mu \mathrm{g} / \mathrm{dL}$ or above. ${ }^{2}$

In 1994, a state government-funded lead management program was established to address this situation. The program (described elsewhere) comprised health promotion, case finding and management, and remediation of contaminated public land. ${ }^{2}$ It was underpinned by an active research and evaluation program. Extensive land remediation work in the highest lead-risk zones was largely completed by 1997 , with final works undertaken in 2003 and 2004. Mining leases adjacent to industrial and public land, including footpaths and vacant blocks, were comprehensively targeted as were some residential blocks considered to pose a hazard; all were within two streets of the mines. Land remediation mainly consisted of covering contaminated soil with an appropriate material (clean soil, clay, mulch, concrete, crushed metal). Where necessary, work was also carried out to prevent storm-water or vehicles from disturbing remediation work. Hardy local native shrubs and grasses were planted in some areas to further stabilise soil and railway trucks transporting lead concentrate were covered.

Since 1991, all 1-4-year-old children in Broken Hill have been offered at least annual blood lead screening. Screening is voluntary; a combination of reminder letters, promotions and advertising in the local media is used to encourage attendance at the lead screening clinic for at least one blood lead test each year.

\section{Methods}

Blood samples are collected for screening at a single laboratory. Trained nurses collect samples by venipuncture 
according to the standard procedure. ${ }^{8}$ Samples are stored at $4{ }^{\circ} \mathrm{C}$ and transported overnight to Adelaide by air for testing the next day. Analysis by electro-thermal atomisation atomic absorption spectrometry is undertaken by the Adelaide Women's and Children's Hospital laboratory.

The number of 1-4-year-old children attending screening each year between 1991 and 2007, their ages, blood lead levels and addresses were obtained from the records of the blood lead screening program. Where children had multiple tests in a year, only the results of the first test were used to avoid regression to the mean. Address was coded into one of five previously described lead-risk zones, based on lead levels in soil (surveyed in 1992) and indoor dust (surveyed in 1995). ${ }^{2}$ Geometric mean soil lead level for the five lead-risk zones (ordered highest to lowest risk) is $1967,794,621,365$ and $262 \mathrm{ppm}$, respectively; geometric mean indoor dust deposition is 946, 717, 490, 216 and $201 \mathrm{mg} / \mathrm{m}^{2}$ per 30 days respectively. ${ }^{2}$

Geometric mean blood lead level (measured as micrograms $(\mu \mathrm{g})$ lead per decilitre $-100 \mathrm{~mL}$ - of blood) was calculated for each lead-risk zone in each year. For comparative purposes these levels were directly age-sex standardised to the 2001 population of 1-4-year-old children. The percentage of children attending for screening was estimated for each lead-risk zone in each year, with estimates of the total eligible population within each zone based on 1991, 1996, 2001 and 2006 census data. ${ }^{9-12}$

Between 1991 and 1999 (after which it was discontinued), a network of dust deposition gauges was maintained in Broken Hill to measure lead flux and concentration. These were constructed and located according to the Australian standard. ${ }^{13}$ The gauges consist of a $150-\mathrm{mm}$-diameter glass funnel inserted through a rubber stopper into a glass bottle of at least 4-L capacity. Gauges are located on stands so that the top of the funnel is approximately $2 \mathrm{~m}$ above ground height and placed in clear areas, away from trees and buildings. The contents of the gauges were collected monthly and all gauges were visited within a few days of each other. The contents were analysed for lead content according to the standard procedure by the Mineral Resources Development Laboratory at Lidcombe, NSW. ${ }^{8}$ For the 17 gauges with data for the period 1991 to 1999 , geometric mean total lead (i.e. water-soluble plus acid-soluble lead reported as mg lead per square metre per month) was calculated for each gauge for each of two periods, 1991-1994 and 1995-1999. Changes in deposited lead across time were assessed by linear regression of yearly averages of $\log _{10}$ transformed total lead against year.

\section{Results}

Between 1991 and 2007, age-sex standardised geometric mean blood lead levels among 1-4-year-olds declined by $65 \%$, from $16.3 \mu \mathrm{g} / \mathrm{dL}$ in 1991 to $5.8 \mu \mathrm{g} / \mathrm{dL}$ in 2007 . Mean blood lead levels of children living in the highest risk zone decreased by $70 \%$, from $27.3 \mu \mathrm{g} / \mathrm{dL}$ in 1991 to $8.3 \mu \mathrm{g} / \mathrm{dL}$ in 2007. Similar declines were experienced in all areas of town until 2001. The average blood lead level of children living in the highest risk zone dropped markedly in 2002, and was similar to that of children living in other areas of town in 2002, 2003, 2005 and 2006, after having been consistently at least $50 \%$ higher (Figure 1). Mean blood lead levels for children living in the highest lead-risk zone spiked in 2004 and again in 2007.

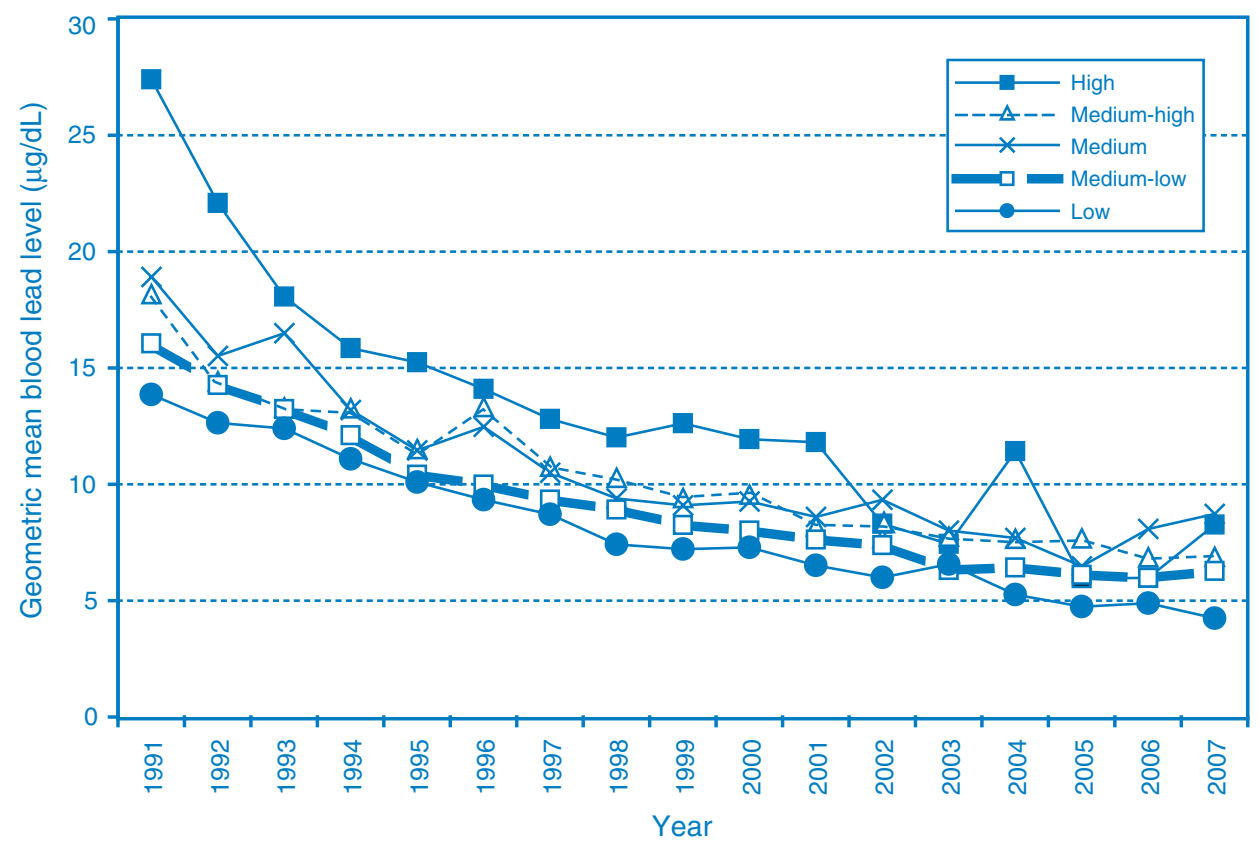

Figure 1. Age-sex standardised geometric mean blood lead levels* for 1-4-year-old children living in different lead-risk** districts of Broken Hill, NSW, 1991-2007. *If a child had multiple blood tests in a year, only the first was used.

**Risk areas based on lead levels in soil and dust. ${ }^{2}$ 
Table 1. Estimated annual participation of 1-4-year-old children in the blood lead screening program in Broken Hill, NSW, 1991-2007, by lead-risk zone

\begin{tabular}{|c|c|c|c|c|c|c|c|c|c|c|c|c|}
\hline \multirow{3}{*}{ Year } & \multicolumn{12}{|c|}{ Number and estimated percent of children } \\
\hline & \multicolumn{2}{|c|}{ High risk* } & \multicolumn{2}{|c|}{ High-medium risk } & \multicolumn{2}{|c|}{ Medium risk } & \multicolumn{2}{|c|}{ Medium-low risk } & \multicolumn{2}{|c|}{ Low risk } & \multicolumn{2}{|c|}{ Overall risk } \\
\hline & $n$ & $\%$ & $n$ & $\%$ & $n$ & $\%$ & $n$ & $\%$ & $n$ & $\%$ & $n$ & $\%$ \\
\hline 1991 & 54 & 69 & 103 & 67 & 105 & 52 & 194 & 48 & 324 & 50 & 780 & 52 \\
\hline 1992 & 51 & 69 & 99 & 68 & 103 & 54 & 171 & 43 & 304 & 48 & 728 & 50 \\
\hline 1993 & 45 & 65 & 76 & 56 & 78 & 43 & 131 & 33 & 207 & 34 & 537 & 39 \\
\hline 1994 & 67 & 104 & 108 & 86 & 128 & 77 & 233 & 61 & 413 & 71 & 949 & 72 \\
\hline 1995 & 46 & 79 & 87 & 77 & 88 & 58 & 216 & 59 & 343 & 63 & 780 & 63 \\
\hline 1996 & 40 & 70 & 71 & 65 & 67 & 47 & 148 & 43 & 242 & 48 & 568 & 49 \\
\hline 1997 & 53 & 96 & 91 & 86 & 81 & 59 & 179 & 51 & 328 & 64 & 732 & 63 \\
\hline 1998 & 56 & 108 & 116 & 117 & 101 & 79 & 199 & 58 & 341 & 69 & 813 & 73 \\
\hline 1999 & 60 & 124 & 90 & 97 & 88 & 72 & 182 & 54 & 313 & 64 & 733 & 67 \\
\hline 2000 & 48 & 104 & 65 & 56 & 79 & 56 & 158 & 49 & 281 & 62 & 631 & 59 \\
\hline 2001 & 45 & 102 & 73 & 62 & 74 & 49 & 142 & 44 & 280 & 65 & 614 & 58 \\
\hline 2002 & 50 & 111 & 79 & 72 & 57 & 39 & 138 & 45 & 257 & 60 & 581 & 56 \\
\hline 2003 & 44 & 94 & 68 & 67 & 69 & 49 & 98 & 33 & 214 & 50 & 493 & 49 \\
\hline 2004 & 39 & 80 & 61 & 64 & 61 & 44 & 132 & 48 & 201 & 46 & 494 & 50 \\
\hline 2005 & 33 & 63 & 71 & 82 & 58 & 44 & 121 & 46 & 228 & 53 & 511 & 53 \\
\hline 2006 & 27 & 48 & 56 & 70 & 50 & 38 & 106 & 43 & 192 & 44 & 431 & 46 \\
\hline 2007 & 30 & 54 & 65 & 81 & 74 & 57 & 89 & 36 & 175 & 40 & 433 & 46 \\
\hline
\end{tabular}

Overall community participation in the blood lead screening program declined from $72 \%$ in 1994 , when there was a major door-knock campaign to recruit children for testing, to 46\% in both 2006 and 2007. Participation remained higher in the highest risk zone (Table 1) but the difference in participation with other zones diminished in 2006 and 2007.

Data from the environmental dust gauges show that, compared with 1991-1994, deposited lead was lower in most gauges during 1995-1999. However, statistically significant reductions only occurred in the three highest risk zones. The three most dusty sites, all of which were in the highest risk zones, showed highly significant reductions in deposited lead, especially after 1997 (Figure 2).

\section{Discussion}

The variable contribution attributed to where a child lives in Broken Hill on their blood lead level has decreased considerably in recent years. Between 1991 and 1993, the average annual difference between highest and lowest locality-specific blood lead level was $9.55 \mu \mathrm{g} / \mathrm{dL}$; this difference was reduced to $4.84 \mu \mathrm{g} / \mathrm{dL}$ during 1994-2001 and to $2.61 \mu \mathrm{g} / \mathrm{dL}$ for 2002-2007 (Figure 1).

Children living in the highest risk zone experienced a sudden reduction in blood lead levels in 2002 that has persisted for four out of six years. The reason for the spikes in blood leads level in 2004 and 2007 is unclear against a background of variable but declining blood lead screening rates. Less than 60 children live in the highest lead-risk zone, which results in some imprecision in estimates for children living in that area. ${ }^{9-12}$ The spikes may be an artefact of the standardisation process: in both 2004 and 2007, 1 - and 2-year-old boys and 4-year-old girls living in the highest risk zone had higher mean blood lead levels than in adjacent years and in other age-sex specific groups; these were also the age and sex groups in that risk zone that had the greatest number of children in the standard population.

The recent differential reduction in blood lead levels observed in children living in the highest lead-risk zone is unlikely to be attributable to changes in screening. Screening participation has declined similarly in all areas, although attendance has remained highest in the higher risk zones, a feature that is consistent with the literature. ${ }^{14,15}$

The decline in blood lead levels for all children in Broken Hill, and particularly those living in the highest risk zone, is consistent with reduced levels of environmental risk. Due to their location, children living closest to the mines would be most likely to benefit from the extensive remediation of contaminated land, the majority of which occurred on the mines and immediately adjacent land and all of which occurred within two streets either side of the mines. Data from the dust deposit gauges (Figure 2) support the 


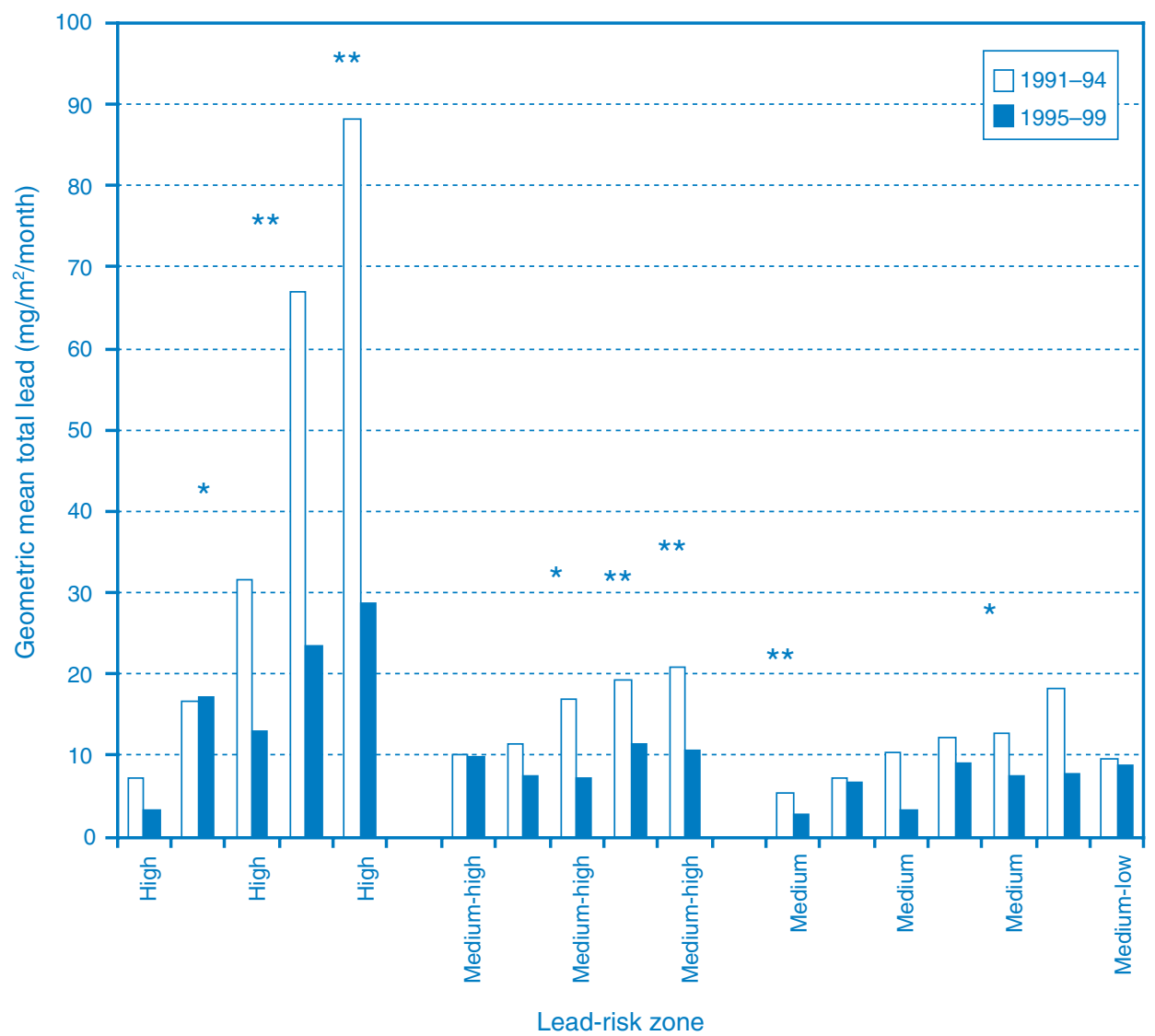

Figure 2. Geometric mean total lead deposition in Broken Hill, NSW, 1991-1999. *Significant at 0.05. ${ }^{* *}$ Significant at 0.01 .

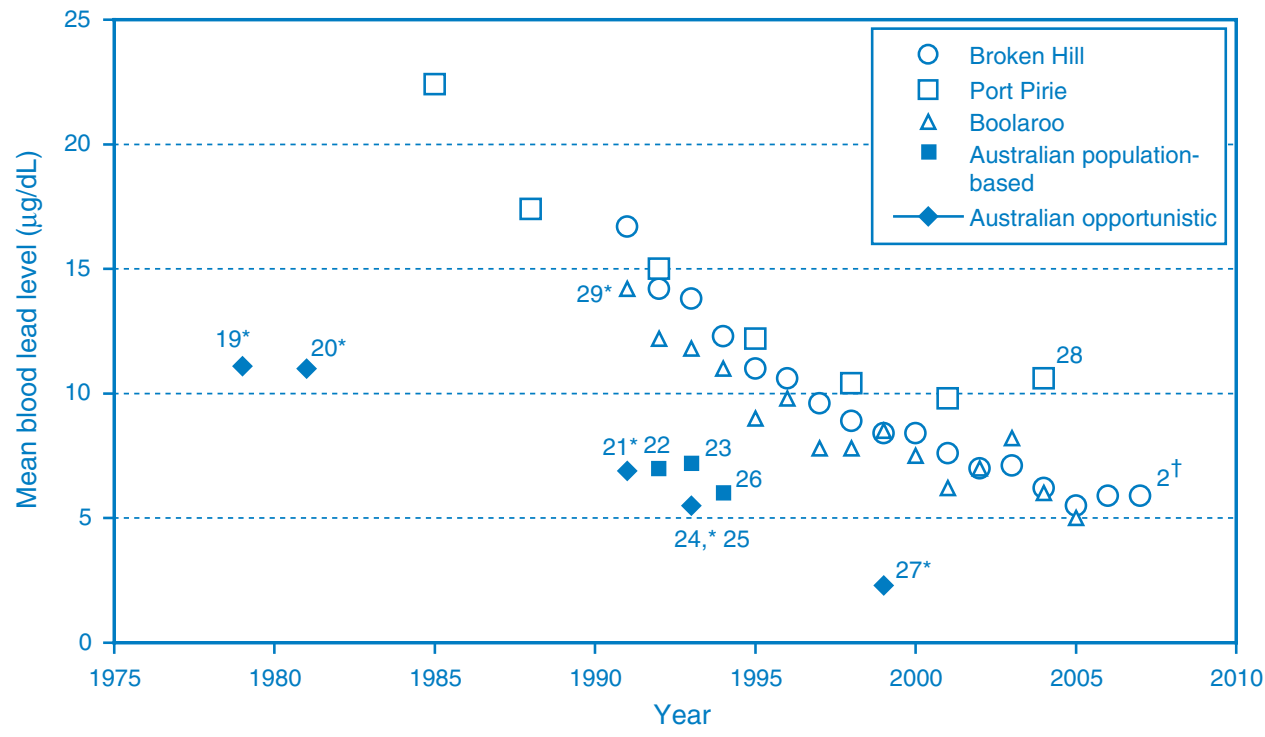

Figure 3. Mean blood lead level of children living in Broken Hill, NSW, compared with other Australian locations. *Indicates arithmetic mean; otherwise geometric. 'Data for Broken Hill 1991-2003 from Lyle et al., 2006; data for 2004-2007 from extended analysis by one of the current authors.

hypothesis that areas closest to the mines experienced the greatest reduction in lead deposition. Sudden and persistent declines in blood lead levels after removal of a source of contamination have been reported from other locations within Australia and overseas. ${ }^{14,16,17}$ In communities where environmental contamination is widespread, zonal remediation is more likely to be effective than intervening in individual homes. ${ }^{17,18}$

The importance of removing environmental risks is highlighted by Figure 3.,19-29 In Australia, lead levels in petrol were lowered in the early 1980s and further reduced in the 
1990s. A number of studies indicate that blood lead levels have reduced markedly in the broader Australian community since the late $1970 \mathrm{~s} .{ }^{19-27}$ While these studies were crosssectional and did not include a remediation assessment component, they provide a benchmark for blood lead levels in young children living outside of point source communities.

While blood lead levels are still substantially above that of the general population, the declines experienced by the three point-source communities of Broken Hill, Port Pirie and Boolaroo, NSW (which had an active lead smelter until 2003) can be attributed to a combination of active local public health programs and broader community-wide action, including reduced lead in petrol and improved control of emissions from mining and smelting. ${ }^{29}$ All three communities experienced similar declines until the late 1990s. Since then, levels in Port Pirie have plateaued at about $10 \mu \mathrm{g} / \mathrm{dL}$ but levels in Broken Hill have continued to decline, probably reflecting the impact of different sources and pathways for lead getting into the environment (an ongoing smelting operation versus contamination from waste dumps and historical smelting). Blood lead levels dropped substantially in Boolaroo after the smelter was closed in $2003 .{ }^{29}$

Thus, after nearly a decade of concerted public health action, we appear to have finally minimised the differential impact of locality on blood lead levels in Broken Hill. Active lead management is still required in the community, and the lead management program is currently being reviewed.

Because of the multifaceted approach taken - a combination of large scale land remediation, intensive case management, including home remediation and health promotion - and the limitations of the service-based data set, including a lack of environmental dust data after 1999, it is not possible to separate the effects of the various components of the program. However, the literature suggests zonal remediation is more likely to be effective in reducing blood lead levels than other interventions. ${ }^{14,16-18}$ The most likely explanation for the important and sustained reduction in blood lead levels among children living in the highest risk zone of Broken Hill is a mitigation of environmental risk by remediation of contaminated land. While we acknowledge that this analysis of available data does not provide definitive evidence of effect, the observation is noteworthy and consistent with the literature.

Combining whole-of-community approaches to public health action with targeted reduction of environmental risks may similarly benefit other communities.

\section{Acknowledgments}

The success of the Broken Hill Lead Management Program reflects the considerable efforts of its staff and community engagement to deal with the problem. Geoffrey Berry undertook analysis of the deposition dust gauge data. The Broken Hill University Department of Rural Health is funded by the Australian Government Department of Health and Ageing.

\section{References}

1. Mark 4:25 in The New American Standard Bible. Available at: http://nasb.scripturetext.com/mark/4.htm (Cited 2 August 2006.)

2. Lyle DM, Phillips AR, Balding WA, Burke H, Stokes D, Corbett S et al. Dealing with lead in Broken Hill: trends in blood lead levels in young children 1991-2003. Sci Total Environ 2006; 359: 111-9. doi:10.1016/j.scitotenv.2005.04.022

3. Thompson AJ. Report of Board appointed to inquire into the prevalence and prevention of lead poisoning at the Broken Hill Silver-Lead Mines. Sydney: Government Printer; 1893.

4. Phillips A. Trends in and factors for elevated blood lead concentration in Broken Hill pre-school children in the period 1991-1993 (dissertation). Newcastle: University of Newcastle; 1998.

5. Woodward-Clyde. Evaluation of environmental lead at Broken Hill. Prepared for Environment Protection Authority, NSW. Project No. 3328. Sydney: AGC Woodward-Clyde Pty Ltd; 1993.

6. Koh TS, Babidge PJ. A comparison of blood lead levels in dogs from a lead mining, lead smelting, urban and rural island environment. Aust Vet J 1986; 63(9): 282-5. doi:10.1111/j.1751-0813.1986.tb08067.x

7. Gulson BL, Yui LA, Howarth D. Delayed visual maturation and lead pollution. Sci Total Environ 1998; 224: 215-9. doi:10.1016/S0048-9697(98)00349-0

8. Standards Association of Australia. Sampling of venous and capillary blood for the determination of lead or cadmium concentration, AS 2636-1988. North Sydney, NSW: Standards Association of Australia; 1988.

9. Australian Bureau of Statistics. CDATA. 1991, CD-ROM. Australian Bureau of Statistics, Canberra.

10. Australian Bureau of Statistics. CDATA. 1996, CD-ROM. Australian Bureau of Statistics, Canberra.

11. Australian Bureau of Statistics. CDATA. 2001, CD-ROM. Australian Bureau of Statistics, Canberra.

12. Australian Bureau of Statistics. 2006 Census of Population and Housing. Cat. No. 2068.0. Canberra: Australian Bureau of Statistics; 2007.

13. Standards Association of Australia. Methods for sampling and analysis of ambient air. Method 10.1. determination of particulates - deposited matter - gravimetric method. AS 3580.10.1. North Sydney, NSW: Standards Association of Australia; 1991.

14. Hilts SR. Effect of smelter emission reductions on children's blood lead levels. Sci Total Environ 2003; 303: 51-8. doi:10.1016/S0048-9697(02)00357-1

15. Kegler MC, Malcoe LH, Kegler SR, Lynch RA, Tolliver R. Caregiver beliefs and behaviours in the prevention of childhood lead poisoning. Fam Community Health 1999; 22(1): $50-65$.

16. Morrison AL. An assessment of the effectiveness of lead pollution reduction strategies in North Lake Macquarie, NSW, Australia. Sci Total Environ 2003; 303: 125-38. doi:10.1016/S0048-9697(02)00353-4

17. Elias RW, Gulson B. Overview of lead remediation effectiveness. Sci Total Environ 2003; 303: 1-13. doi:10.1016/S00489697(02)00361-3 
18. Sheldrake S, Stifelman M. A case study of lead contamination cleanup effectiveness at Bunker Hill. Sci Total Environ 2003; 303: 105-23. doi:10.1016/S0048-9697(02)00354-6

19. De Silva PE, Donnan MB. Blood lead levels in Victorian children. Med J Aust 1981; 1(1): 93.

20. Rathus M, Latham S, Golding G, Rowan C. Blood lead levels in Queensland children. Med J Aust 1982; 2: 183-5.

21. Threlfall T, Kent N, Garcia-Webb P, Byrnes E, Psaila-Savona P Blood lead levels in children in Perth, Western Australia. Aust J Public Health 1993; 17(4): 379-81.

22. Mira M, Bawden-Smith J, Causer J, Alperstein G, Karr M, Snitch P et al. Blood lead concentrations of preschool children in Central and Southern Sydney. Med J Aust 1996; 164: 399-402.

23. Cowie C, Black D, Fraser I. Blood lead levels in preschool children in eastern Sydney. Aust N Z J Public Health 1997; 21(7): 755-61

24. Taylor R, Bazelmans J, Golec R, Oakes S. Declining blood lead levels in Victorian children. Aust J Public Health 1995; 19(5): 455-9.
25. Aldrich R, Toneguzzi R, Wlodarczyk J, Hensley M, Nichols B, Gruszynski $\mathrm{C}$ et al. Opportunistic blood lead testing in a paediatric inpatient population. Aust N Z J Public Health 1997; 21(2): 163-7. doi:10.1111/j.1467-842X.1997.tb01677.x

26. Australian Institute of Health and Welfare. Lead in Australian children: Summary of the national survey of lead in children. Canberra: Australian Institute of Health and Welfare; 1995.

27. Public Health Division. The health of the people of New South Wales - Report of the Chief Health Officer 2000. Sydney: NSW Department of Health; 2000.

28. Maynard EJ, Franks LJ, Malcolm MS. The Port Pirie Lead Implementation Program: Future Focus and Direction. Adelaide: Department of Health South Australia; 2006.

29. Dalton CB, Bates LI. Impact of closure of a large lead-zinc smelter on elevated blood lead levels of children in adjacent suburbs, Boolaroo, Australia. WIT Transact Ecology Health 2005; 85: 377-87. Available at: http://library.witpress.com/ pages/PaperInfo.asp?PaperID=15811 (Cited 28 August 2008.) 